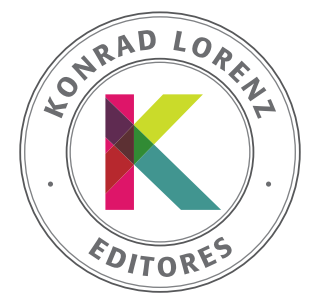

\title{
Estrés percibido y felicidad en adultos mexicanos según estado de salud-enfermedad
}

\author{
Ania Chávez-Amavizcaa, Julia Gallegos-Guajardoa*, María del Rocío Hernández-Pozo ${ }^{\mathrm{b}}$, Jeanette \\ López-Wallec, Cecilia Castor-Pragac, María Araceli Álvarez-Gascab ${ }^{b}$, Cecilia Meza-Peñac ${ }^{c}$, Tania \\ Romo-González ${ }^{\mathrm{d}}$, Raquel González-Ochoa ${ }^{\mathrm{d}}$ y Elías Alfonso Góngora-Coronadoe
}

\author{
a Universidad de Monterrey, México \\ b Universidad Nacional Autónoma de México, México \\ c Universidad Autónoma de Nuevo León, México \\ ' Universidad Veracruzana, México \\ e Universidad Autónoma de Yucatán, México
}

Recibido el 13 junio de 2019; aceptado el 11 marzo de 2020

\author{
PALABRAS CLAVE \\ Estrés percibido, \\ felicidad, \\ enfermedad, \\ salud, \\ adultos mexicanos
}

\section{KEYWORDS}

Perceived stress, happiness, disease, health, mexican adults

\begin{abstract}
Resumen El presente estudio tuvo como objetivo analizar la relación entre el estrés percibido y la felicidad en adultos mexicanos, así como comparar ambas variables según el estado de salud-enfermedad. La muestra estuvo conformada por 762 adultos mexicanos con una media de edad de 44.96 años, de los cuales el 70.3 \% reportó no padecer ninguna enfermedad. Se utilizó la Escala de Estrés Percibido y el Inventario de Felicidad Auténtica. Adicionalmente, los participantes reportaron si padecían alguna enfermedad y seleccionaron, posteriormente, en una lista de opciones. Mediante el SPSS v.24 se realizaron análisis de frecuencias, descriptivos, análisis de correlación y análisis de varianza de un factor. En los resultados se observó una correlación negativa y estadísticamente significativa entre el estrés percibido y la felicidad. Por su parte, solo se observaron diferencias significativas en la subescala de estrés percibido "sobre-pasado por la situación"; fue mayor en el grupo de personas con una enfermedad aguda en comparación con el grupo de personas sanas. Se discuten los principales hallazgos, así como las implicaciones para la práctica psicológica.

(c) 2020 Fundación Universitaria Konrad Lorenz. Este es un artículo Open Access bajo la licencia CC BY-NC-ND (http://creativecommons.org/licenses/bync-nd/4.0/).
\end{abstract}

Perceived stress and happiness among Mexican adults split by health-illness condition

Abstract The present study aimed to analyze the relationship between perceived stress and happiness in Mexican adults, and test for differences in both variables according to the health-disease condition. The sample consisted of 762 Mexican adults with an average age of 44.96 years, of which $70.3 \%$ reported not suffering from any disease. The Perceived Stress Scale

\footnotetext{
* Autor para correspondencia.

Correo electrónico: julia.gallegos@udem.edu
} 
and the Authentic Happiness Inventory were used. In addition, participants reported if they suffer from any disease, later selecting from a list of options. Using the SPSS v.24, frequency, descriptive, correlation and analysis of variance of one factor were performed. Negative and statistically significant correlation between perceived stress and happiness was found. On the other hand, only significant differences were observed in the subscale of perceived stress "overwhelmed by the situation" being greater in the group of people with an acute illness, compared to the group of healthy people. The main findings as well as implications for psychological practice are discussed.

(c) 2020 Fundación Universitaria Konrad Lorenz. This is an open access article under the CC BYNC-ND license (http://creativecommons.org/licenses/bync-nd/4.0/).

La relación entre el estrés psicológico y los indicadores de salud física ha sido ampliamente estudiada y documentada en investigaciones que muestran cómo altos niveles de estrés psicológico se asocian con mayor riesgo a padecer ciertas enfermedades, y con el deterioro del estado de salud física y percibida (Furman, Joseph \& Miller-Perrin, 2018; Heruti, Levy \& Avitsur, 2018; Reed, Sejunaite \& Osborne, 2016; Weekes, MacLean \& Berger, 2005). De forma más reciente se ha documentado una relación entre las llamadas emociones positivas y diferentes aspectos de salud física y salud percibida, de modo que se propone que estas tienen un efecto "protector" de las enfermedades y promueven el estado de salud (Howell, Kern \& Lyubomirsky, 2007; Lazarus, 2009; Piqueras, Ramos, Martínez \& Oblitas, 2009). Aunque en México existe una creciente línea de investigación que describe variables positivas, como, por ejemplo, la felicidad y el bienestar en los mexicanos (Palomar, 2005; Rojas, 2005), son escasos los estudios que las analizan en relación con la salud física en esta población. El presente estudio pretende contribuir a reducir esta brecha en la investigación.

Las principales causas de muerte en adultos a nivel mundial se relacionan con aspectos de salud-enfermedad, según señala la Organización Mundial de la Salud (OMS, 2016). En el 2016, las principales causas de mortalidad a nivel mundial fueron enfermedades crónicas no transmisibles (ENT), reportadas como las más prevalentes la cardiopatía isquémica y el accidente cerebrovascular (OMS, 2016). En México, según datos del Instituto Nacional de Estadística, Geografía e Informática (Inegi), durante el 2017 las principales causas de muerte en adultos fueron problemas relacionados con la salud $(88.6 \%)$; las causas más prevalentes fueron las enfermedades del corazón (20.1\%), la diabetes mellitus (15.2\%) y los tumores malignos (12.0\%), todas estas enfermedades crónicas no transmisibles. Las ENT se caracterizan por tener una larga duración, en su mayoría, y porque resultan de la combinación de diversos factores de riesgo de tipo genético, fisiológico, ambiental y conductual (OMS, 2014). Además de representar las principales causas de muerte en adultos a nivel mundial y en México, las ENT afectan la calidad de vida y el bienestar psicológico de quién las padece y de sus familiares (OMS, 2014); de ahí la relevancia de estudiar factores psicológicos asociados.

El estrés psicológico es resultado de eventos ambientales, la percepción de dichos eventos y la manera en que la persona los afronta (Lazarus, 2009). De acuerdo con Lazarus (2009), la valoración o la percepción de amenaza juega un papel mediador entre los eventos ambientales y la reacción de estrés fisiológico. Estos pensamientos evaluativos han sido denominados appraisal y coping ("evaluación" y "afrontamiento"): appraisal en cuanto es la evaluación del significado de lo que está sucediendo y de la cual depende el coping, que se describe como un proceso de esfuerzos continuos de la persona en pensamiento y acción dirigidos a manejar demandas específicas evaluadas como abrumadoras (Lazarus, 2009). Desde este enfoque cognitivo, al grado en el cual las situaciones en la vida de una persona se evalúan como estresantes se le conoce como "estrés percibido" (Cohen, Kamarck \& Mermelstein, 1983).

El estrés percibido puede afectar la salud mental y física cuando una persona se percibe sobrepasada por una situación, y cuando este nivel de estrés se mantiene en el tiempo (Lazarus, 2009; McEwen \& Stellar, 1993). Weekes et al. (2005) encontraron que el nivel de estrés percibido puede funcionar como factor de riesgo para la aparición de síntomas físicos. Los autores condujeron un estudio con una muestra de universitarios estadounidenses $(N=107,65$ mujeres, 42 hombres, en un rango de edad de 18 a 21 años) con el objetivo de explorar la relación entre la exposición a los estresores, el estrés percibido y síntomas de salud física negativos tales como fatiga extrema, reacciones alérgicas, tos, fiebre y resfriados o malestar estomacal, entre otros indicadores que formaron parte del inventario desarrollado por los investigadores. Dentro de los principales hallazgos se observó que las mujeres reportaron tasas estadística y significativamente más altas que los hombres por molestias y factores estresantes. Asimismo, tanto en hombres como en mujeres la exposición al estrés se asoció de forma significativa con problemas de salud, y solo en las mujeres el estrés percibido y los problemas de salud se correlacionaron de forma significativa. Finalmente, en ambos géneros la exposición a estresores y la percepción de estrés predijeron los problemas de salud, en un $58 \%$ el primero y en $44 \%$ el segundo (Weekes et al., 2005).

Por otra parte, Furman et al. (2018) encontraron que el estrés percibido puede interferir en conductas y hábitos de salud física. Los autores condujeron una investigación con 131 estudiantes estadounidenses con una media de edad de 19 años, y observaron correlaciones positivas y significativas entre el estrés percibido y la baja calidad del sueño evaluada con el Pittsburgh Sleep Quality Index (el cual contiene subescalas de calidad del sueño subjetiva, latencia del sueño, duración del sueño, eficiencia habitual del sueño, trastornos del sueño, uso de medicamentos para el sueño y disfunción diurna). Sin embargo, no encontraron 
una relación entre el estrés percibido y los indicadores de salud física (presión arterial nocturna). De forma similar, Reed et al. (2016) observaron relaciones entre el estrés percibido y los síntomas de salud física. Los autores exploraron la relación entre el estrés percibido y los síntomas de salud física y la calidad de vida relacionada con la salud con una muestra británica de 122 madres de familia de niños diagnosticados con síndrome del espectro autista. En los resultados se observó que el estrés percibido se correlacionó negativa y significativamente con la calidad de vida relacionada con la salud de forma positiva con los síntomas de salud física negativa (Reed et al., 2016). Las diferencias observadas en los resultados del estudio de Furman et al. (2018) y el estudio de Reed et al. (2016) podrían deberse a que se utilizaron diferentes muestras: en el primer estudio se trabajó con una muestra comunitaria, mientras que en el segundo estudio se trabajó con una muestra en riesgo. Como señalan Fernández y Edo (1994), las emociones influyen en el estado de salud a través de diferentes mecanismos, como, por ejemplo, al afectar los hábitos de salud de tal modo que impactan la salud física.

Con respecto al estudio de la felicidad en relación con el proceso de salud-enfermedad, diversas investigaciones sustentan una relación positiva y significativa entre las emociones positivas y la salud física (Fredrickson, 2013; Fredrickson \& Joiner, 2018; Kyriopoulos, Athanasakis \& Kyriopoulos, 2018; Lazarus, 2009). Las emociones positivas se asocian con la activación del sistema nervioso parasimpático, lo cual produce estados de relajación e inhibición de estrés (Fredrickson, 2013; Lazarus, 2009). Según Seligman (2008), existen dos razones principales para ocuparse del tema de la felicidad en relación con la salud: la primera es que los seres humanos desean bienestar por sí mismo, más allá de aliviar su sufrimiento; la segunda es que trabajar sobre el bienestar puede resultar una de las mejores estrategias para tratar el desorden mental y los estados mentales que funcionan como factores de riesgo para la salud. Entre las llamadas emociones positivas se encuentra la felicidad subjetiva, definida a partir de un componente emocional-afectivo y un componente cognitivo o de valoración respecto a las propias circunstancias de vida (Diener, Emmons, Larsen, Griffin, 1985; Lyubomirsky \& Lepper, 1997).

Diversas investigaciones han documentado que estados emocionales positivos tales como la felicidad y el bienestar subjetivo favorecen el estado de salud (Kok et al., 2013; Kyriopoulos et al., 2018; Howell et al., 2007; Piqueras et al., 2009). Howell et al. (2007) llevaron a cabo un metaanálisis con el objetivo de analizar si las emociones positivas favorecen el estado de salud de las personas, para lo cual consideraron una gama de 150 estudios que incluían mediciones de felicidad, satisfacción con la vida, afecto positivo y optimismo. Entre los principales hallazgos encontraron que el bienestar subjetivo estaba relacionado positiva y significativamente con los resultados de salud a corto plazo, a largo plazo y con el control de la enfermedad o los síntomas, lo que, de acuerdo con los autores, permite argumentar que el bienestar puede reforzar de forma directa el funcionamiento inmune y amortiguar el impacto del estrés (Howell et al., 2007). Un estudio conducido posteriormente por Kok et al. (2012), realizado con 65 adultos estadounidenses ( $M=37.5$ años, 66 \% mujeres, 34 \% hombres), buscó comprobar el vínculo entre las emociones positivas y la salud física (para esto utilizó el tono vagal como indicador), mediado por las percepciones de las personas de sus conexiones sociales positivas. Los principales hallazgos de este estudio sugieren que las emociones positivas, las conexiones sociales positivas y la salud física se influyen mutuamente (Kok et al., 2012).

Hallazgos de otros estudios muestran que la salud física también se relaciona con incrementos en la felicidad subjetiva (Gerstenbluth, Rossi \& Triunfo, 2008; Lobos, Mora, Del-Carmen, Caligaria \& Schnettlerd, 2015). Lobos et al. (2015) condujeron un estudio con 1163 adultos chilenos divididos en dos grupos: adultos jóvenes con una media de edad de 28 años, y adultos maduros con una media de edad de 50.6 años. Esto con el objetivo de identificar factores de salud que determinan la felicidad, como, por ejemplo, una pobre percepción del estado de salud y el número de días no saludables, encontrando que estos son fuertes predictores de la felicidad (Lobos et al., 2015). Por su parte, Gerstenbluth et al. (2008) encontraron en población argentina y uruguaya que el buen estado de salud aumentó la probabilidad de ser feliz en ambos países.

En cuanto a la relación entre el estrés percibido y la felicidad subjetiva, diversas investigaciones han documentado una relación inversa y significativa (Hernández \& Landero, 2014; McMahan et al. 2016; Schiffrin \& Nelson, 2010). Schiffrin y Nelson (2010) llevaron a cabo un estudio con una muestra de 100 estudiantes universitarios estadounidenses cuyos resultados mostraron una correlación negativa y significativa entre el estrés percibido y la felicidad subjetiva. Por su parte, McMahan et al. (2016) condujeron un estudio con 180 universitarios coreanos y encontraron también una correlación inversa y significativa entre el estrés percibido y la felicidad subjetiva. En México, Hernández y Landero (2014) encontraron en una muestra clínica de mujeres con cáncer de mama ( $N=90$, edades entre 36 y 76 años) correlaciones negativas y significativas entre la percepción del estado de salud y el estrés percibido, y entre el estrés percibido y la felicidad; asimismo, encontraron una correlación positiva y significativa entre la felicidad y la percepción del estado de salud.

En México existen antecedentes que describen variables positivas tales como la felicidad y el bienestar en los mexicanos (Palomar, 2005; Rojas, 2005), y se ha encontrado que el estrés percibido y la felicidad se relacionan de forma negativa en muestras clínicas (Hernández \& Landero, 2014). Sin embargo, actualmente no existen estudios que describan y analicen estas variables en una muestra general en función del estado de salud. El objetivo del presente estudio fue describir el estrés percibido y la felicidad en adultos mexicanos, analizar su relación e identificar si existen diferencias según el estado de salud-enfermedad. A partir de la literatura se plantearon las siguientes hipótesis: (1) el estrés percibido y la felicidad mantienen una relación inversa y significativa en adultos mexicanos; (2) existen diferencias significativas en la variable estrés percibido entre grupos según el estado de salud-enfermedad; (3) existen diferencias significativas en la variable felicidad entre grupos según el estado de salud-enfermedad.

\section{Método}

\section{Participantes}

El estudio tuvo un diseño transversal, no experimental, de alcance correlacional. La muestra estuvo conformada por 762 adultos mexicanos con un rango de edad de 18 a 
76 años $(M=44.96$ años, $D E=8.86$ años, $37.4 \%$ hombres y $62.6 \%$ mujeres), residentes de nueve estados de la República Mexicana (Nuevo León, $n=321$; Veracruz, $n=167$; Ciudad de México, $n=138$; Estado de México, $n=48$; Yucatán, $n=36$; Oaxaca, $n=24$; Puebla, $n=24$; Hidalgo, $n=3$; y Quintana Roo, $n=1)$. El $70.3 \%$ reportó no padecer ninguna enfermedad (538 casos), mientras que el resto (224 casos) reportó padecer alguna enfermedad.

\section{Instrumentos}

La información referente al estado de salud-enfermedad se obtuvo a través de dos preguntas cerradas: a la primera pregunta los participantes respondieron de forma afirmativa o negativa si padecían alguna enfermedad; aquellos que contestaron de forma afirmativa a la primera pregunta se les solicitó posteriormente elegir entre una lista de opciones el tipo de enfermedad que padecían.

\section{Escala de Estrés Percibido (EEP) (Cohen, Kamarck \& Mermelstein, 1983)}

El estrés percibido se evaluó con la versión mexicana de la EEP (González \& Landero, 2007). La EEP se creó con el propósito de medir el grado en que los eventos de la vida se consideran estresantes. En su versión mexicana, la EEP consta de 14 ítems con opción de respuesta tipo Likert de 0 (nunca) a 4 (muy frecuente), y ha mostrado una buena consistencia interna $(a=.83)$ (González \& Landero, 2007). La EEP-14 tiene dos tipos de reactivos, aquellos que hacen referencia a sentirse "en control de la situación" y aquellos que hacen referencia a "sentirse sobrepasado por la situación".

\section{Inventario de Felicidad Auténtica (IFA-24) (Seligman, Steen, Park \& Peterson, 2005)}

A fin de medir la felicidad se utilizó la versión en español del Authentic Happiness Inventory (ahí) (Seligman et al., 2005), el cual se diseñó para medir estados afectivos positivos caracterizados por el disfrute, la gratificación y el propósito o sentido en la vida. El IFA consta de 24 reactivos con opción de respuesta tipo Likert de 1 a 5 . En muestra mexicana, el Grupo CO-EDUCA (Hernández-Pozo et al., en prensa) ha encontrado una estructura de dos factores con una consistencia interna alta-aceptable (factor 1, $a=.947$; factor 2, $a=.779$ ).

\section{Procedimiento y análisis de datos}

El presente estudio forma parte de un proyecto más amplio del Grupo Co-Educa (Hernández-Pozo et al., 2019). A fin de obtener el consentimiento informado de cada participante del estudio se siguieron procedimientos estándar. El mismo formato se utilizó en electrónico y en papel, y fue el mismo que cada persona firmó aceptando participar de forma voluntaria y a través del cual se informó sobre el objetivo y la duración del estudio, las indicaciones generales y la garantía de confidencialidad en el tratamiento de sus respuestas, así como la especificación de riesgos y los beneficios de participar. Los participantes se contactaron a través de psicólogos colaboradores del grupo de investigación distribuidos en diferentes partes del país, quienes invitaron a personas dentro de su círculo de interacciones frecuentes a que respondieran de forma voluntaria una encuesta en línea o de manera presencial.
El análisis de datos se realizó con el Paquete Estadístico para las Ciencias Sociales (SPSS, v. 24). Respecto al estado de salud-enfermedad, se generaron cinco categorías en función de la presencia-ausencia de enfermedad y del grado de molestias e impacto psicológico que cada tipo de enfermedad tiene en razón a su nivel de gravedad. Se realizaron los siguientes análisis: análisis de frecuencias por tipo de enfermedad; análisis descriptivos para las variables de estrés percibido y felicidad; análisis de correlación bivariada con las variables felicidad, estrés percibido, estrés percibido "en control de la situación" y estrés percibido "sobrepasado por la situación"; finalmente, se realizó el análisis de varianza (Anova) de un factor, con la finalidad de identificar diferencias entre grupos a partir del tipo de enfermedad en las variables felicidad, estrés percibido, estrés percibido "en control de la situación" y estrés percibido "sobrepasado por la situación”.

\section{Resultados}

El objetivo del presente estudio fue describir el estrés percibido y la felicidad en adultos mexicanos, analizar su relación e identificar si existen diferencias según el estado de salud-enfermedad. Las frecuencias de casos por estado de salud-enfermedad se muestran en la tabla 1, en la que se observa que, del total de la muestra, el $70.6 \%$ reportó estar sano (a), el $14.4 \%$ reportó padecer alguna enfermedad crónica indolora, el 7 \% reportó padecer alguna enfermedad aguda, el $4.3 \%$ reportó padecer alguna enfermedad crónica dolorosa o crónica fulminante, y el 3.7 \% reportó alguna enfermedad psicosomática, adicción o no especificada. Por otra parte, respecto a la variable de estrés percibido $(M=1.47, D E=0.64)$, de forma general se identificó en la muestra que el 31.9 \% (243 casos) se ubicó en un rango bajo, el 53.3 \% (406 casos) se ubicó en rango medio, y el 14.8 \% (113 casos) se ubicó en rango alto. En cuanto a la variable felicidad $(M=3.21, D E=0.66)$, se identificó que el 20.7 \% de la muestra se ubicó en rango bajo (157 casos), el $53.9 \%$ se ubicó en rango medio (411 casos) y el 25.5\% (194 casos) se ubicó en rango alto. En la tabla 1 se muestran los estadísticos descriptivos para las variables estrés percibido y felicidad, según estado de salud-enfermedad.

Respecto a la relación entre el estrés percibido y la felicidad, los resultados del análisis de correlación mostraron una relación negativa y estadísticamente significativa en la muestra $(r=-.614, p<.01)$. En específico, el factor "en control de la situación" de la EEP-14 se correlacionó de forma positiva y estadísticamente significativa con la felicidad $(r=.591$, $p<.01$ ), mientras que el factor "sobrepasado por la situación” se correlacionó de forma negativa y estadísticamente significativa con la felicidad $(r=-.496, p<.01)$. En la tabla 2 se muestran los resultados del análisis de correlación.

Respecto al análisis de diferencias entre grupos según el estado de salud-enfermedad en las variables estrés percibido y felicidad, los resultados del análisis de varianza (Anova) de un factor mostraron, principalmente, diferencias en la subescala "sobrepasado por la situación". Se observaron diferencias significativas entre grupos en la variable estrés percibido $(F[4]=3.596, p=.006)$, sin embargo, al realizar las pruebas post hoc utilizando estadístico de Bonferroni, solo se observaron diferencias significativas entre los grupos de personas sanas y los que reportaron padecer alguna enfermedad aguda $(p=.030)$. En específico, 
Tabla 1 Descriptivos para felicidad y estrés percibido según estado de salud-enfermedad

\begin{tabular}{|c|c|c|c|c|c|c|}
\hline Estado de salud-enfermedad & Frecuencia & Porcentaje & Media EEP-14 & $\mathrm{DE}$ & Media IFA & DE \\
\hline Total & 762 & $100 \%$ & 1.47 & 0.64 & 3.21 & 0.66 \\
\hline Sano $=0$ & 538 & $70.6 \%$ & 1.42 & 0.63 & 3.24 & 0.65 \\
\hline $\begin{array}{r}\text { Aguda }=1 \\
\text { trastorno digestivo } \\
\text { infecciosa } \\
\text { inflamatoria } \\
\text { respiratoria } \\
\text { sistema urinario } \\
\text { anemia } \\
\text { trastorno menstrual }\end{array}$ & 53 & $7 \%$ & 1.73 & 0.52 & 3.00 & 0.73 \\
\hline $\begin{array}{r}\text { Psicosomática, adicción y no especificada }=2 \\
\text { adicción } \\
\text { trastorno alimentario } \\
\text { trastorno emocional } \\
\text { restricción perceptual } \\
\text { dos o más } \\
\text { otra }\end{array}$ & 28 & $3.7 \%$ & 1.64 & 0.87 & 2.99 & 0.85 \\
\hline $\begin{array}{r}\text { Crónica indolora }=4 \\
\text { cardio } \\
\text { hipertensión } \\
\text { endócrina } \\
\text { hepática } \\
\text { diabetes }\end{array}$ & 110 & $14.4 \%$ & 1.43 & 0.59 & 3.23 & 0.66 \\
\hline $\begin{array}{r}\text { Crónica dolorosa y crónica fulminante }=5 \\
\text { gota } \\
\text { neurológica } \\
\text { osteo-articular } \\
\text { fibromialgia } \\
\text { lupus } \\
\text { osteomielitis } \\
2 \text { cáncer } \\
\text { HIV-SIDA }\end{array}$ & 33 & $4.3 \%$ & 1.60 & 0.67 & 3.08 & 0.64 \\
\hline
\end{tabular}

Nota: EEP-14= Escala de Estrés percibido; IFA= Inventario de Felicidad Auténtica.

Tabla 2 Análisis de correlación con las variables de estrés percibido y felicidad

\begin{tabular}{lcccc}
\hline & 1 & 2 & 3 & 4 \\
\hline 1. EEP-14 & 1 & $-.862^{* *}$ & $.895^{* *}$ & $-.614^{* *}$ \\
2. EEP-14, F1, en control & - & 1 & $.545^{* *}$ & 1 \\
3. EEP-14, F2, sobrepasado & - & - & $-.496^{* *}$ & 1 \\
4. IFA & - & - & 1 \\
\hline
\end{tabular}

Nota: ** la correlación es significativa al 0.01 bilateral $(p<.01)$. EEP-14 = Escala de Estrés Percibido; IFA= Inventario de Felicidad Auténtica.

los resultados del Anova mostraron diferencias significativas entre grupos en la subescala "sobrepasado por la situación” de la variable estrés percibido $(F[4]=4.023, p=.003)$; al realizar las pruebas post hoc, solo se observaron diferencias significativas entre el grupo de personas sanas y el grupo de personas que reportaron padecer alguna enfermedad aguda $(p=.010)$. Por otra parte, los resultados del Anova no mostraron diferencias significativas entre grupos en la subescala “en control de la situación" de la variable estrés percibido $(F[4]=1.775, p=.132)$. Finalmente, aunque los resultados del Anova mostraron diferencias significativas entre grupos en la variable felicidad $(F[4]=2.662, p=.032)$, no se observaron diferencias significativas entre grupos en las pruebas post hoc. Los resultados del análisis de varianza de las variables de estrés percibido y felicidad, según estado de salud-enfermedad, se muestran en la tabla 3. 
Tabla 3 ANOVA de un factor: Estrés percibido y felicidad según estado de salud-enfermedad

\begin{tabular}{|c|c|c|c|c|c|c|c|c|c|c|c|c|}
\hline Grupos & EEP & $F$ & $p$ & $E E P(1)$ & $F$ & $p$ & $E E P(2)$ & $F$ & $p$ & IFA & $F$ & $p$ \\
\hline Total & 1.47 & 3.59 & 0.006 & 2.87 & 1.775 & 0.132 & 1.82 & 4.023 & 0.003 & 3.21 & 2.62 & 0.032 \\
\hline 0 & 1.42 & & & 2.91 & & & 1.77 & & & 3.24 & & \\
\hline 1 & 1.73 & & & 2.71 & & & 2.18 & & & 3.00 & & \\
\hline 2 & 1.64 & & & 2.73 & & & 2.02 & & & 2.99 & & \\
\hline 4 & 1.43 & & & 2.85 & & & 1.80 & & & 3.23 & & \\
\hline 5 & 1.60 & & & 2.73 & & & 1.94 & & & 3.08 & & \\
\hline
\end{tabular}

Nota: EEP-14 = Escala de Estrés Percibido; EEP (1)= Escala de Estrés Percibido “en control de la situación”; EEP (2)= Escala de Estrés Percibido "sobrepasado por la situación"; IFA= Inventario de Felicidad Auténtica; F= estadístico F de Fisher-Snedecor; $p=$ nivel de significancia estadística.

\section{Discusión}

El presente estudio buscó comprobar dos hipótesis: la primera, que el estrés percibido y la felicidad mantienen una relación inversa y significativa en adultos mexicanos; la segunda que se planteó es que existen diferencias significativas en la variable estrés percibido y en la variable felicidad entre grupos según el estado de salud-enfermedad. Los hallazgos de este estudio permiten confirmar parcialmente estas hipótesis.

A partir de los resultados del análisis de correlación se asume que existe una relación negativa y estadísticamente significativa entre el estrés percibido y la felicidad en población general de adultos mexicanos. Esto coincide con los hallazgos encontrados por Hernández y Landero (2014) en una muestra clínica en México, y coincide con lo encontrado en estudios previos con muestras de otros países (McMahan et al., 2016; Schiffrin \& Nelson, 2010). A partir de estos hallazgos podemos argumentar que en población general mexicana existe una tendencia a experimentar menor nivel de felicidad (entendido en términos de experiencias afectivas satisfactorias, con propósito y con significado) entre mayor sea el nivel de estrés percibido, y viceversa: cuanto mayor sea el nivel de felicidad menor será el nivel de estrés percibido. Esto tiene implicaciones para la práctica psicológica, tal como lo han sugerido los teóricos: trabajar aspectos positivos como, por ejemplo, la felicidad, puede amortiguar el impacto del estrés, así como reforzar de forma directa el funcionamiento del sistema inmunológico (Howell et al., 2007, Lazarus, 2009). Debido a lo anterior resulta recomendable incluir en el tratamiento psicológico del estrés los objetivos y las técnicas vinculadas a la evaluación y la promoción del bienestar subjetivo y la felicidad.

Por otra parte, la correlación positiva y estadísticamente significativa que se observó entre estrés percibido "en control de la situación" y felicidad, y la correlación negativa y estadísticamente significativa que se observó entre estrés percibido "sobrepasado por la situación" y felicidad, coincide con lo planteado por Lazarus (2009) con respecto a la interdependencia entre el estrés y las emociones positivas, es decir, que en la presente muestra de adultos mexicanos el estrés que se experimenta cuando se asume el control de la situación es el que mantiene una interdependencia con la felicidad, mientras que, cuanto mayor sea el estrés experimentado por una persona que se asume sobrepasada por la situación, menor será su nivel de felicidad.
Con relación a la variable estado de salud-enfermedad, en los resultados del presente estudio se observó que el grupo con enfermedades crónicas (indoloras, no-fulminantes) tuvo una mayor prevalencia con relación al resto de los grupos por tipo de enfermedad, lo cual coincide con lo reportado a nivel mundial (OMS, 2016) y a nivel nacional (Inegi, 2018). Al comparar entre grupos la variable de estrés percibido solo se observaron diferencias significativas entre el grupo de personas sanas y el grupo de personas con enfermedades agudas, en particular en la subescala "sobrepasado por la situación". Estos resultados sugieren que ante la presencia de una enfermedad aguda el nivel de estrés percibido será mayor, lo cual coincide parcialmente con lo encontrado por otros investigadores (Reed et al., 2016; Weekes et al., 2005). Una posible explicación para estos resultados podría ser que, como señalan Frankenhaeuser y Stokols, Novaco, Stokols y Campbell (como se citan en Taylor, 2007), cualquier situación amenazante en un principio producirá estrés, pero tal reacción se elimina con el paso del tiempo, es decir, la mayoría de las personas es capaz de adaptarse psicológicamente a algunos estresores. Dado que las enfermedades agudas tienen un comienzo súbito y una evolución rápida, esto podría generar en la persona la valoración de estar sobrepasada por la situación; en cambio, las enfermedades crónicas son de progresión lenta y de larga duración (OMS, 2019), lo cual permitiría a la persona asimilar los cambios derivados de la enfermedad y desplegar recursos para afrontar o adaptarse al proceso.

Respecto a la comparación de grupos con la variable felicidad, las diferencias observadas no fueron estadísticamente significativas, por lo cual no se puede asumir que el nivel de felicidad en adultos mexicanos varíe significativamente según el estado de salud-enfermedad. Estos resultados discrepan de lo reportado en otras investigaciones, cuyos principales hallazgos han vinculado positiva y significativamente la felicidad con los resultados de salud a corto y largo plazo, y con el control de enfermedades o síntomas (Howell et al., 2007); asimismo, han planteado que un cambio adverso en la salud reduce la satisfacción con la vida, y cómo cuanto peor es el cambio en salud mayor es la reducción de la satisfacción con la vida (Easterlin, 2003).

Posibles explicaciones a los hallazgos del presente estudio en relación con el nivel de felicidad según el estado de salud-enfermedad podrían encontrarse al seguir el modelo de tres factores para explicar la felicidad de Lyubomirsky, Sheldon y Schkade (2005). El primer factor es el set point (punto fijo), a partir del cual una persona experimenta 
felicidad, es relativamente estable en el tiempo y lo proporciona la genética y las características de personalidad: al parecer los niveles de felicidad y bienestar subjetivo en México son altos en comparación con otros países, pues según el reporte de resultados del Happy Planet Index (HPI, 2019) México se ubica en el lugar número 11 de 140 en el nivel de bienestar subjetivo. El segundo factor es circumstances ("circunstancias"), el cual explicaría cambios en el nivel de felicidad dados por factores circunstanciales a los que la persona se adapta después de un tiempo, volviendo al punto fijo para experimentar felicidad; en este sentido, una posible explicación a los hallazgos del presente estudio podría ser que existe una adaptación casi total o total de los participantes a los cambios de salud. Finalmente, el tercer factor es intentional activity (actividad intencionada). Incluye una variedad de cosas que las personas piensan y hacen en su vida cotidiana, tales como realizar ejercicio, revaluar las situaciones desde una perspectiva más optimista o las relaciones sociales que establecen. En este sentido, Lamu y Oslen (2017) encontraron en adultos con enfermedad crónica que la mejoría en el estado de salud tiene efectos en el bienestar subjetivo a través de las relaciones sociales, por tanto, el deterioro de las condiciones de salud afectaría el bienestar subjetivo al afectar la interacción o vinculación de la persona con su red social. Esto último pone de relevancia la necesidad de estudiar otras variables, como, por ejemplo, el apoyo social en la relación salud-felicidad en adultos mexicanos.

\section{Conclusión}

El presente estudio confirmó que existe una relación negativa y significativa entre el estrés percibido y la felicidad en adultos mexicanos. Asimismo, se confirmó la existencia de diferencias significativas en la variable estrés percibido, en particular la subescala "sobrepasado por la situación", solo entre el grupo de personas sanas y el grupo de personas con una enfermedad aguda. Esto indica la importancia de tomar en cuenta el factor de tiempo de evolución de la enfermedad al explorar esta relación. Como hallazgo inesperado no se encontraron diferencias estadísticamente significativas por grupo de salud-enfermedad en la variable de felicidad, por lo que se sugiere seguir indagando sobre este tema y estimar el tamaño del efecto de estas diferencias.

\section{Agradecimientos}

Agradecemos el apoyo financiero parcial por parte del proyecto PAPIIT DGAPA UNAM IG300415, del cual fueron responsables la tercera y sexta autoras, para la realización del estudio.

\section{Referencias}

Cohen, S., Kamarck, T., \& Mermelstein, R. (1983). A global measure of perceived stress. Journal of Health and Social Behavior, 24(4), 385-396. https://doi.org/10.2307/2136404

Diener, E., Emmons, R. A., Larsen, R. J., \& Griffin, S. (1985). The satisfaction with life scale. Journal of Personality Assessment, 49(1), 71-75. https://doi.org/10.1207/s15327752jpa4901_13

Easterlin, R. (2003). Explaining happiness. Proceedings of the $\mathrm{Na}$ tional Academy of Sciences of the United States of America, 100(19), 11176-11183.
Fernández, J., \& Edo, S. (1994). Emociones y salud. Anuario de Psicología, 61, 25-32.

Fredrickson, B. (2013). The value of positive emotions. American Scientist, 91(4), 330-335.

Fredrickson, B., \& Joiner, T. (2018). Reflections on positive emotions and upward spirals. Perspectives on Psychological Science, 13(2), 194-199. https://doi.org/10.1177/1745691617692106

Furman, M., Joseph, N., \& Miller-Perrin, C. (2018). Associations between coping strategies, perceived stress, and health indicators. Psi Chi Journal of Psychological Research, 23(1), 61-91. https://doi.org/10.24839/2325-7342.jn23.1.61

Gerstenbluth, M., Rossi, M., \& Triunfo, P. (2008). Felicidad y salud: una aproximación al bienestar en el Río de la Plata. Estudios de Economía, 35(1), 65-78.

González, M., \& Landero, R. (2007). Factor structure of the Perceived Stress Scale (PSS) in a sample from Mexico. The Spanish Journal of Psychology, 10, 199-206. https://doi.org/10.1017/ s1138741600006466

The Happy Planet Index. (2019). México. Recuperado de http:// happyplanetindex.org/countries/mexico

Hérnandez-Moreno, F., \& Landero, R. (2014). Propiedades psicométricas de la Escala de Felicidad Subjetiva y su relación con el estrés, la salud percibida y el apoyo social en pacientes con cáncer de mama. Psicooncología, 11(2-3), 357-367. https://doi. org/10.5209/rev_psic.2014.v11.n2-3.47394

Hernández-Pozo, M. R., López-Walle, J. M., Álvarez-Gasca, M. A., Romo-Gonzalez, T., Gallegos-Guajardo, J., Meza-Peña, C., \& Muñoz-López, M. A. (en prensa). Niveles de estrés percibido en función de características sociodemográficas y de salud. Manuscrito enviado a dictamen para su publicación.

Heruti, I., Levy, S., \& Avitsur, R. (2018). Physical injury, health, and well-being: Role of stress perception. Injury, 49(8), 1546-1551. https://doi.org/10.1016/j.injury.2018.06.022

Howell, R., Kern, M., \& Lyubomirsky, S. (2007). Health benefits: meta-analytically determining the impact of well-being on objective health outcomes. Health Psychology Review, 1(1), 83136. https://doi.org/10.1080/17437190701492486

Instituto Nacional de Estadística, Geografía e Informática. (2018). Características de las defunciones registradas en México durante 2017. Recuperado de https://bit.ly/34y9xnL

Kok, B., Coffey, K., Cohn, M., Catalino, L., Vacharkulksemsuk, T.. Algoe, B., \& Fredrickson, B. (2013). How positive emotions build physical health: Perceived positive social connections account for the upward spiral between positive emotions and vagal tone. Psychological Science. 24(7) 1123-1132.

Kyriopoulos, I., Athanasakis, K., \& Kyriopoulos, J. (2018). Are happy people healthier? An instrumental variable approach using data from Greece. Journal of Epidemiology and Community Health, 72, 1153-1161. https://doi.org/10.1136/jech-2018-210568

Lamu, A., \& Olsen, J. (2017). Yes, health is important, but as much for its importance via social life: The direct and indirect effects of health on subjective well-being in chronically ill individuals. Health Economics, 27, 209-222. https://doi. org/10.1002/hec. 3536

Lazarus, R. (2009). Estrés y emoción. Manejo e implicaciones en nuestra salud. España: Editorial Desclée de Brouwer.

Lyubomirsky, S., \& Lepper, H. (1997). A measure of subjective happiness: preliminary reliability and construct validation. Social Indicators Research, 46(2), 137-155.

Lyubomirsky, S., Sheldon, K., \& Schkade, D. (2005). Pursuing happiness: the architecture of sustainable change. Review of $\mathrm{Ge}$ neral Psychology, 9(2), 113-131.

Lobos, G., Mora, M., Del Carmen Lapo, M., Caligaria, C., \& Schnettler, B. (2015). Happiness and health and food-related variables: evidence for different age groups in Chile. Suma Psicológica, 22(2), 120-128. https://doi.org/10.1016/j.sumpsi.2015.09.002 
McEwen, B., \& Stellar, E. (1993). Stress and the individual. Mechanisms leading to disease. Archives of Internal Medicine, 153(18), 20932101. https://doi.org/10.1001/archinte.1993.00410180039004

McMahan, E., Choi, I., Kwon, Y., Choi, J., Fuller, J., \& Josh, P. (2016). Some implications of believing that happiness involves the absence of pain: negative hedonic beliefs exacerbate the effects of stress on well-being. Journal of Happiness Studies, 17(6), 2569-2593. https://doi.org/10.1007/s10902-0159707-8

Organización Mundial de la Salud. (2014). Informe sobre la situación mundial de las enfermedades no transmisibles. Recuperado de https://bit.ly/2PKgSMF

Palomar, J. (2005). Estructura del bienestar subjetivo: construcción de una escala multidimensional. En L. Garduño, B. Salinas, \& M. Rojas (Eds.), Calidad de vida y bienestar subjetivo en México (pp. 113-142). México: Plaza y Valdés.

Piqueras, J., Ramos, V., Martínez, A., \& Oblitas, L. (2009). Emociones negativas y su impacto en la salud mental y física. Suma Psicológica, 16(2), 85-112.

Reed, P., Sejunaite, K., \& Osborne, L. A. (2016). Relationship between self-reported health and stress in mothers of children with autism spectrum disorders. Journal of Autism and Developmental Disorders, 46(3), 934-941. https://doi.org/10.1007/ s10803-015-2638-8
Rojas, M. (2005). El bienestar subjetivo en México y su relación con indicadores objetivos. Consideraciones para la política pública. En L. Garduño, B. Salinas \& M. Rojas (Eds.), Calidad de vida y bienestar subjetivo en México (pp. 83-111). México: Plaza y Valdés.

Seligman, M. E. P., Steen, T. A., Park, N., \& Peterson, C. (2005). Positive psychology progress: empirical validation of interventions. American Psychologist, 60(5), 410-421. https://doi. org/10.1037/0003-066x.60.5.410

Seligman, M. (2008). Positive health. Applied Psychology, 57(1), 3-18. https://doi.org/10.1111/j.1464-0597.2008.00351.x

Schiffrin, H., \& Nelson, S. (2010). Stressed and happy? Investigating the relationship between happiness and perceived stress. Journal of Happiness Studies, 11(1), 33-39. https://doi.org/10.1007/ s10902-008-9104-7

Taylor, S. (2007). Estrés. En S. Taylor (Ed.), Psicología de la salud (pp. 153-211). México: McGraw-Hill.

Weekes, N., MacLean, J., \& Berger, D. (2005). Sex, stress, and health: does stress predict health symptoms differently in the two sexes? Stress and Health, 21(3), 147-156. https://doi. org/10.1002/smi.1046 